\title{
The effect of maze structure upon the performance of a multiple-goal task
}

\author{
JOHN HORNER \\ Duke University, Durham, North Carolina
}

\begin{abstract}
The ability of rats to find food in three kinds of mazes (radial, parallel, antiradial) was studied. Each maze had eight goalboxes. All goals were baited at the beginning of a trial, and the animal's task was to retrieve food from all eight goals. The task was divided into two phases. In the first phase, the animal was allowed access to four randomly chosen goalboxes. Once the animal had visited all four of the goals, the second phase of the trial began, in which the animal was allowed access to all eight goalboxes. The animals showed a strong initial tendency to avoid already visited goalboxes in the radial maze, but not in the parallel maze. In the antiradial maze, the animals showed a strong initial tendency to avoid already visited goalboxes during the first phase of the trial, but not in the second phase. Significant improvement in performance over sessions was seen only in groups that did not have a strong initial tendency to avoid already visited goalboxes. These data suggest that maze structure influences performance by producing different initial patterns of exploration that may be either appropriate or inappropriate to the multiple-goal task.
\end{abstract}

In a multiple-goal task (MGT), a number of goals are baited at the onset of each trial and the reward is not replenished when removed. The MGT requires that an animal get food from all goals while avoiding goals it has already visited. Recent work has shown that rats easily learn to perform this task (Olton \& Samuelson, 1976; Olton \& Schlosberg, 1978). Olton and his associates (Olton, 1978, 1979; Olton \& Schlosberg, 1978) have suggested that the ease with which rodents learn this task is a result of natural "win-shift" foraging strategy, that is, a tendency to avoid a recently sampled food site. An alternative explanation has been advanced by Gaffan and Davies (1981). They suggested that the animal's performance on the MGT is related to how appropriate its exploratory behavior is to the requirements of the task. For example, Gaffan and Davies (1981, Experiment 3) eliminated the animal's initial tendency to alternate between goal arms and found that the animals had greater difficulty performing on a MGT. The present study explored whether an animal's performance on a MGT in different mazes depends upon its initial pattern of entries into goal arms.

The tendency to alternate spontaneously between goal arms is a behavior that is appropriate to performance of the MGT. The tendency to alternate spontaneously is strong regardless of whether or not food is found in the goal arm (Gaffan \& Davies, 1981) or whether or not the animals are food deprived (Dennis, 1935). Spontaneous alternation occurs in both multiple-choice mazes (Dennis \&

This research was supported by a grant from the National Science Foundation to J. E. R. Staddon. The author's mailing address is: Department of Psychology, Duke University, Durham NC 27706.
Sollenberger, 1934; Gaffan \& Davies, 1981) and single-choice mazes (Dennis, 1935). Spontaneous alternation occurs regardless of whether the animal is returned to the choice point by the experimenter (Dennis, 1935) or returns on its own (Dennis \& Sollenberger, 1934). The tendency to spontaneous alternation is weakened by the animal's familiarity with the choices offered (Gaffan \& Davies, 1981) and by the animal's being allowed to consume only part of the reward found in the goal (Herrmann, Bahr, Bremner, \& Ellen, 1982).

Spontaneous alternation is highly dependent upon maze configuration (Douglas, Mitchell, \& Kentala, 1972; Jackson, 1941). Jackson was able to relate the strength of spontaneous alternation to the angular separation of the goals. He found that when the angular distance between two goals is zero (i.e., parallel arms), the probability that an animal will enter a given arm is independent of the previous choice. Douglas et al. (1972), in a similar study, found that for reliable spontaneous alternation to occur, the two choices must end in "spatially distinct" goals.

The general hypothesis presented in this paper is that the animal's performance on a MGT depends upon factors that influence the animal's pattern of entries into goals - such as partial feeding, familiarity, or maze structure. Specifically, the paper deals with how different maze structures affect the animal's initial pattern of entries into goal arms, and hence its performance on the MGT. For example, the most frequently used multiple-goal maze is the radial maze: a central platform and eight symmetrical arms, each ending in a goalbox (Figure 1). Animals trained in this maze quickly learn not to reenter goalboxes. The radial maze is ideal for spontaneous 


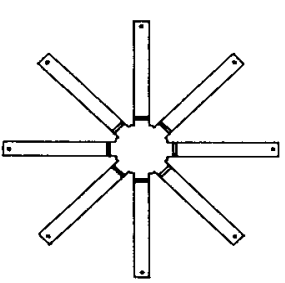

RADIAL

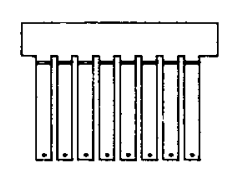

PARALLEL

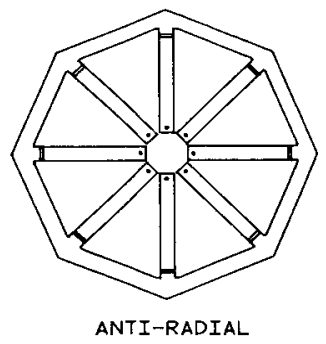

Figure 1. Diagram of radial, parallel, and antiradial mazes. The dots represent the goal points. Doors are represented by two lines drawn across the alley.

alternation because it has spatially distinct goalboxes and goal arms separated as widely as possible. If spontaneous alternation is appropriate to a MGT, then the animal should perform better in a radial maze-which favors spontaneous alternation-than in a maze that does not favor spontaneous alternation. Dale (1982) has recently reported a study in which rats reentered previously visited arms more often in an eight-arm parallel maze (Figure 1) than in a comparable eight-arm radial maze. The parallel maze is not ideal for spontaneous alternation because of its spatially contiguous goalboxes and samedirection goal arms.

In all multiple-goal single-choice mazes, such as the radial and parallel mazes, the occurrence or nonoccurrence of spontaneous alternation will determine whether an animal's pattern of entries is appropriate or inappropriate to the MGT. However, in one type of maze-the multiple-goal multiple-choice mazespontaneous alternation can be made inappropriate to the MGT. The antiradial maze (Figure 1) favors spontaneous alternation because of the angular separation between goal arms and peripheral alley. But in this maze, spontaneous alternation will produce entries into any encountered goal arm, regardless of whether the arm has been entered previously.

The rationale for this phenomenon is illustrated in Figure 2. When the rat is first placed in the antiradial maze, before the doors to the goal arms are opened, it invariably makes one or more circuits of the peripheral alley, thus visiting the peripheral segments A and B. Suppose the animal is moving from segment $A$ to segment $B$ in the periphery (Figure 2) when the alley door to goal arm $C$ is opened. When confronted with a choice of arms A, B, or C, the animal should choose the goal alley, $C$, because it has never visited that alley before. When the rat leaves the goal arm, C, it should choose the outer alley, B, because that outer alley is the one the animal has visited least recently-since it came to the chbice point initially from peripheral segment $A$ and has just been down goal arm $\mathrm{C}$. This pattern of choices will drive the animal in one direction around the maze, entering every arm it encounters. As long as the animal does not miss a goal alley, this is an efficient strategy. However, if the animal does miss one, or if the ani- mal has already visited some of the arms, the tendency to alternate spontaneously at each choice point gets it into trouble. For example, when the animal again approaches choice $\mathrm{ABC}$ from outer alley $\mathrm{A}$, spontaneous alternation again dictates that it enter goal arm C, because that choice will again constitute the least recently visited alley. The tendency to enter any encountered goal arm can be either appropriate or inappropriate to performance on the multiple-goal task, depending upon whether or not this tendency leads to entries into previously visited goal arms.

The present experiment compared performance in the radial, antiradial, and parallel mazes on the forced choice of the first four arms/choose four remaining arms (force 4/choose 4) task used by Olton and Schlosberg (1978). In the first phase of this experiment, the animal was allowed access to four randomly chosen goal arms. Once it had entered all four goal arms, the second phase of the trial was begun. In the second phase, the animal was allowed access to all eight goal arms.

Our hypothesis predicted that the animals run in the radial maze should do well during both phases of the experiment, because the animals' tendency to alternate goal arms spontaneously is appropriate to this MGT. Animals run in the parallel maze, however, should do poorly in both phases, because their tendency to reenter already visited goal arms is inappropriate to this MGT. Animals run in the antiradial maze should do well in the first phase, but not in the second phase, of the experiment. This is because, in the first phase, the tendency to enter every encountered arm in order around the maze ensures that the animal will enter all open arms without reentering

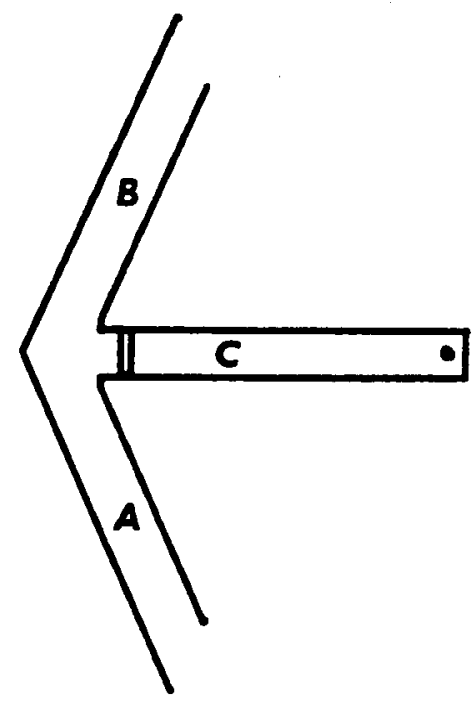

Figure 2. A single choice point in an antiradial maze. $A$ and $B$ represent two segments of the peripheral alley. $C$ represents the goal arm. The expected pattern of entry is to travel from $A$ to $C$ to $B$ regardless of any previous entries into the goalbox. See text for explanation. 
previously visited arms-thus being appropriate to this MGT. In the second phase, however, the tendency to enter every encountered goal arm in order around the maze will lead to errors, and thus is inappropriate to this MGT. The hypothesis does not predict anything about the amount of each group's improvement in the MGT over sessions, except to say that animals that start out doing well in the MGT have less room for improvement than do those that start out doing poorly in the MGT. The hypothesis does imply that if groups that have patterns of entries that are inappropriate to performance of the MGT are to improve their performances, they must change their patterns of entries to be appropriate to the task.

\section{METHOD}

\section{Subjects}

Twenty-four male Long-Evans rats between 120 and 140 days of age were used. The animals were housed in individual cages and exposed to continuous light. Water was provided at all times. Approximately 10 days before training began, all the animals were placed on a restricted diet of Purina Laboratory Chow and weights were recorded daily. When the animals were reduced to $75 \%$ of ad-lib body weight, they were fed daily amounts sufficient to maintain this weight. Prior to training, all the animals had at least 4 days with their weights at $75 \%$ of ad lib. On the day before training began, the animals were fed entirely on $45-\mathrm{mg}$ Noyes food pellets.

\section{Apparatus}

All three of the mazes used-radial, parallel, and antiradialhad eight enclosed arms. In each maze, the floor and the walls of the alley were constructed of plywood; the roof of each maze was covered with hardware cloth, thus allowing the animal a view of the room. All alleys were $11.5 \mathrm{~cm}$ wide, and their walls were $16.5 \mathrm{~cm}$ high; the goal alleys were $83 \mathrm{~cm}$ long. The floor of each maze was covered with $.5 \mathrm{~cm}$ of San-i-cel. The goalboxes were eight plywood platforms elevated $2.5 \mathrm{~cm}$ above the alley floor. Each platform had a small indentation that was $1 \mathrm{~cm}$ in diameter and $.5 \mathrm{~cm}$ deep. Each goalbox was $72 \mathrm{~cm}$ from the entrance to the goal arm. Access to the arms was controlled by a guillotine door $2.5 \mathrm{~cm}$ from the entrance. Each door could be manually controlled by wires.

In the radial maze, the central platform was $36 \mathrm{~cm}$ in diameter and served as the starting and ending box for the animals in this maze. The parallel maze startbox was $21.5 \times 128 \mathrm{~cm}$; one of the long sides bordered the entrances to the eight goal arms. The antiradial maze had no startbox; entrance into this maze was made via one of the outside alleys. The entrances into the larms of the antiradial maze were $82 \mathrm{~cm}$ apart.

The mazes were placed in a well-lighted room that contained numerous pieces of equipment (tables, etc.). A video camera mounted in the ceiling was used to observe the animals' behavior. The experimenter sat behind a screen while each animal was run.

\section{Procedure}

The animals were divided randomly into three groinps of eight. Each group was run in only one maze. The sessions were run on 12 consecutive days, one session per day. Before the beginning of every session, each goal was baited with two 45-mg Noyes food pellets. At the start of the session, the animal was placed in the start location. All guillotine doors were closed, so that the animal was contained either within the startbox (radial and parallel maze) or in the outer alleys (antiradial maze). After $15 \mathrm{sec}_{\text {, four of the }}$ guillotine doors were raised and the animal was allowed to choose among four open goal arms. These four goal arms were counterbalanced such that no three adjacent arms were open during this phase and all goal arms were open equally often over the 12 sessions of the experiment. When the animal had retrieved all food from the four open goal arms in this preconfinement phase, he was confined to the start location by the lowering of all the doors. When a 15-sec confinement period had elapsed, all eight doors were raised. During this postconfinement phase, the animal could choose among all eight goal arms. The session was terminated when all remaining goal arms were entered, or when eight goal arms were entered during the postconfinement phase, or when $12 \mathrm{~min}$ had elapsed since the start of the trial.

The arms entered and the time of entry were recorded. Also, data on whether the animal retrieved the food in the goal and the time of confinement between the two phases were kept. From these data, the pattern of entries was broken down into three categories. The first pattern, termed immediate reentry, occurred when an animal entered a goal arm that it had already visited without entering another goal arm in the interim. This type of entry was predicted for the parallel group because the design of the maze does not favor the occurrence of spontaneous alternation. The second pattern, termed unidirectional, occurred when an animal entered the next open goal arm, but always in the same direction from the last arm it had entered. This type of pattern was predicted for the antiradial group. The third type of pattern was one in which the animal showed neither an immediate reentry nor a unidirectional tendency, and this was referred to as a typical pattern. Because the rats showing a unidirectional pattern require only four entries to complete the preconfinement phase, only the first four entries during a phase were used to assign a pattern of entries to a category. Trials in which animals did not complete the phase in question, as defined above, were not categorized.

\section{RESULTS}

The hypothesis presented in this paper predicts three things. First, the patterns of entries observed in the different mazes can be predicted from what is known about how maze structure affects an animal's tendency to alternate among the arms of a maze. Second, the animal's performance on the MGT depends upon whether the initial pattern of entries is appropriate or inappropriate to the task. Third, any improvement in the animal's performance on the MGT must be paralleled by a change in the animal's pattern of entries to be appropriate to the taisk.

The initial patterns of entries into goalboxes differed depending upon which maze the animal was trained in. Table 1 presents data on the pattern of entries during the preconfinement phase for Session 2. Table 1 shows that rats trained in the parallel maze showed a higher relative frequency of immediate reentries than did rats in either the radial or antiradial mazes. Table 1 also shows that rats trained in the antiradial maze were more likely to show a unidirectional pattern than those rats trained in either the radial or parallel mazes. The radial group produced more "typical" patterns because, unlike the animals in the parallel maze, the radial group was avoiding already visited boxes; and, unlike animals in the antiradial maze, they were not necessarily using a simple response rule, such as the unidirectional pattern. 
A chi-square test shows that the differences in Table 1 were significant at the $2 \%$ level $\left[\chi^{2}(4)=13.14\right]$. The results of the chi-square reveal only that there was a significant difference in the table; the chi-square does not give a quantitative measure of the level of the reduction in error achieved in predicting pattern of entry by knowing the type of maze the animal was trained in. Thus, a nonparametric measure of predictive value, Goodman and Kruskal's lambda (Mueller, Schuessler, \& Costner, 1970), was also applied to the data. Lambda by columns (using the maze structure as the independent variable) shows that the reduction in error achieved by knowing what maze the animals were trained in was $33 \%$.

Table 2 presents data on the pattern of entries during the postconfinement phase for Session 2. Although included in Table 2, the parallel group during the postconfinement phase was not included in this analysis because the number of animals completing this phase for Session 2 was too small (i.e., $\mathrm{N}=3$ ). A chisquare test shows that the differences in Table 2 were significant at the $1 \%$ level $\left[\chi^{2}(1)=7.27\right]$. Lambda by columns shows that the reduction in error achieved by knowing what maze the animal was trained in was $40 \%$.

Our hypothesis predicts that the animal's performance on the MGT depends upon whether the pattern of entries is appropriate or inappropriate to the task. Figure 3 shows the average number of prevously unvisited goal arms entered per group during the first four entries in the preconfinement phase. Data points were averaged over two sessions, making the total possible number of trials, averaged, 16 (8 animals $\times 2$ sessions). The radial and antiradial

Table 1

The Pattern of Entry by Maze During the Preconfinement Phase for Session 2

\begin{tabular}{|c|c|c|c|c|c|c|}
\hline \multirow[b]{3}{*}{ Pattern } & \multicolumn{6}{|c|}{ Mazes } \\
\hline & \multicolumn{2}{|c|}{ Radial } & \multicolumn{2}{|c|}{ Antiradial } & \multicolumn{2}{|c|}{ Parallel } \\
\hline & Obs & $\operatorname{Exp}$ & Obs & Exp & Obs & Exp \\
\hline Typical & 6 & 3.83 & 2 & 3.83 & 3 & 3.35 \\
\hline Unidirectional & 2 & 2.43 & 5 & 2.43 & 0 & 2.13 \\
\hline Immediate Reentry & 0 & 1.74 & 1 & 1.74 & 4 & 1.52 \\
\hline
\end{tabular}

Note-Obs = observed $;$ Exp $=$ expected.

Table 2

The Pattern of Entry by Maze During the Postconfinement Phase for Session 2

\begin{tabular}{|c|c|c|c|c|c|c|}
\hline \multirow[b]{3}{*}{ Pattern } & \multicolumn{6}{|c|}{ Mazes } \\
\hline & \multicolumn{2}{|c|}{ Radial } & \multicolumn{2}{|c|}{ Antiradial } & \multicolumn{2}{|c|}{ Parallel } \\
\hline & Obs & Exp & Obs & Exp & Obs & $\operatorname{Exp}$ \\
\hline Typical & 8 & 5.5 & 3 & 5.5 & 1 & \\
\hline Unidirectional & 0 & 2.5 & 5 & 2.5 & 0 & \\
\hline Immediate Reentry & 0 & & 0 & & 2 & \\
\hline
\end{tabular}

Note-Obs = observed $;$ Exp $=$ expected.

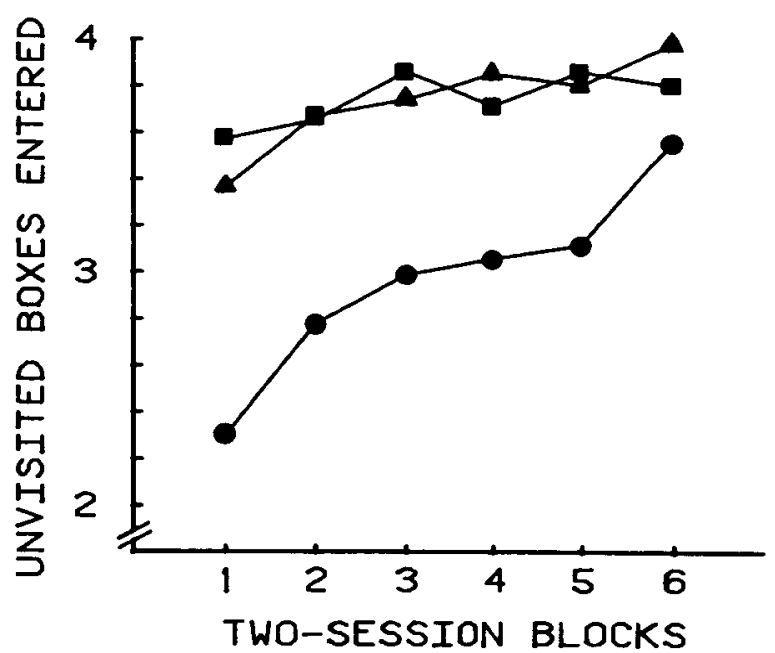

Figure 3. The average number of new boxes entered during the first four entries in the preconfinement phase for two-session blocks. The radial group is represented by closed squares $(\square)$, the parallel group by closed circles $(\bullet)$, and the antiradial group by closed triangles $(\Delta)$.

groups, in which the initial pattern of entries was appropriate to the task in the preconfinement phase, both did significantly better during the first four sessions at entering previously unvisited goal arms than did the parallel group $(z=5.61, p<.01$, radial vs. parallel group; $z=5.19, p<.01$, antiradial vs. parallel group-two-tailed Mann-Whitney U test). There was no significant difference between the radial and antiradial groups in the first four sessions of the experiment $(\mathrm{z}=.87, \mathrm{p}>.05$, radial vs. antiradial group -two-tailed Mann-Whitney U test).

Figure 4 shows the average number of previously unvisited goal arms entered per group during the first four entries in the postconfinement phase. Points not represented for the parallel group indicated an averaged two-session block in which fewer than 10 trials out of a possible 16 were completed. The combined score of the parallel group for any 1 day was never greater than 2.25 and ranged between 1.25 and 2.25 .

During the first four sessions, the radial group entered significantly more previously unvisited goal arms in the postconfinement phase than did the parallel group ( $z=3.38, p<.01$ - two-tailed Mann-Whitney $\mathrm{U}$ test). The radial group also did significantly better during the first four sessions at entering previously unvisited goal arms in the postconfinement phase than did the antiradial group $(z=3.27, p<.01-$ two-tailed Mann-Whitney U test).

If the animals in the antiradial group used a unidirectional pattern in the postconfinement phase, they should make entries into every arm they encountered. This is inappropriate to the MGT and would result in the animals' entering 2.5 previously unvisited goal arms during the first four entries. This expected performance was calculated using a Monte 


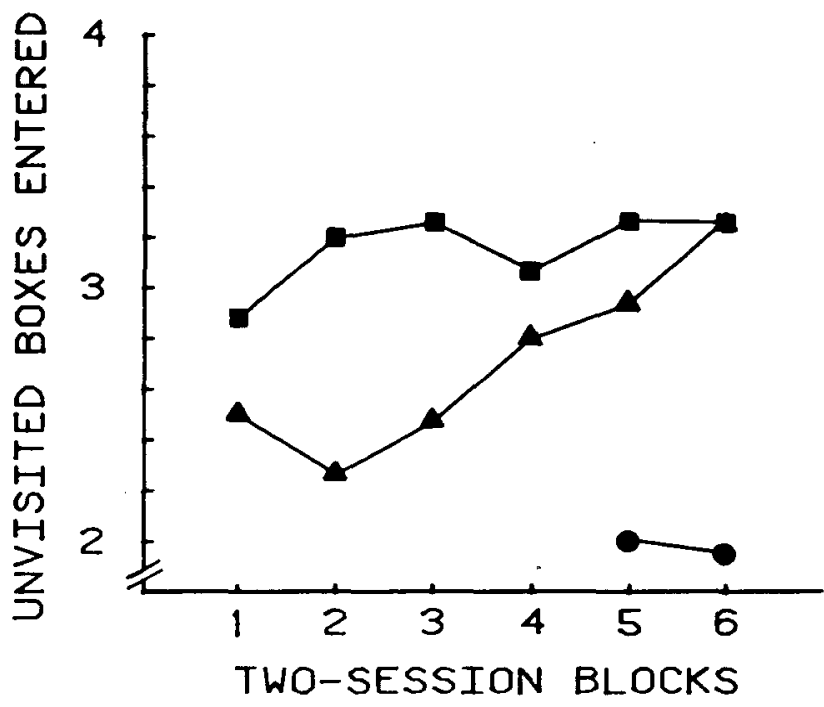

Figure 4. The average number of new boxes entered during the first four entries in the postconfinement phase for two-session blocks. The radial group is represented by closed squares (E), the parallel group by closed circles $(\bullet)$, and the antiradial group by closed triangles $(\Delta)$. A data point is not represented if a group had fewer than 10 completed trials out of a possible 16 over the two-session block being averaged.

Carlo method. In a simulated maze, four of eight goals were designated as already visited, using the counterbalancing methods discussed in the procedure. The first goal arm entered and the direction the simulated animal took were chosen at random. Then the number of previously unvisited boxes entered was determined by counting the number of boxes designated as previously unvisited from the first goal arm entered to the next three in the chosen direction. The performance of the antiradial group from Sessions 1 through 6, as seen in Figure 4, was close to 2.5 .

The animal's initial performance on the MGT was related to whether its initial pattern of entries was appropriate or inappropriate to the MGT. The amount of improvement in performance on the MGT is not predicted by the present hypothesis, except that those animals that start out doing well have less room for improvement than those that start out doing poorly.

Figures 3 and 4 show that all groups improved their performance over sessions. Whether the improvements seen in Figures 3 and 4 are statistically significant is yet another question. The radial group did not show a significant improvement in either the pre- or postconfinement phases $\left(T=3, N_{s-r}=5, p>.05\right.$, Session 2 vs. Session 12, preconfinement; $T=7$, $\mathrm{N}_{\mathrm{s}-\mathrm{r}}=6, \mathrm{p}>.05$, Session 2 vs. Session 12, postconfinement-Wilcoxon sign test). The antiradial group did not show a significant improvement in the preconfinement phase, but did show a significant improvement in the postconfinement phase $(T=3$,
$\mathrm{N}_{\mathrm{s}-\mathrm{r}}=5, \mathrm{p}>.05$, Session 2 vs. Session 12 , preconfinement phase; $T=0, N_{s-r}=7, p<.05$, Session 2 vs, Session 12, postconfinement phase-Wilcoxon sign test). The parallel group showed significant improvement in the preconfinement phase but not in the postconfinement phase $\left(T=0, N_{s-r}=6, p<.05\right.$, Session 2 vs. Session 12, preconfinement phase; $T=0, N_{s-r}=4$, $\mathrm{p}>.05$, Session 9 vs. Session 12, postconfinement phase-Wilcoxon sign test). Session 9 was chosen for comparison of the parallel group in the postconfinement phase because it was the first session in which all the animals in the parallel group completed this phase. Even though the Wilcoxon sign test did not indicate significant improvement in the postconfinement phase for the parallel group, improvement in performance can be seen in the increase in the number of animals completing this phase over sessions. The number of animals in the parallel group completing the postconfinement phase in the first session was one, whereas the number completing this phase in the ninth session was eight.

In all these results except one-the parallel group in the postconfinement phase-those animals that initially had patterns of entries that were inappropriate to the MGT showed a significant improvement in performance, whereas those that initially had patterns of entries that were appropriate to the MGT did not show a significant improvement in performance. It is certainly feasible that had the experiment been continued for more sessions, the parallel group would also have shown a significant improvement in performance during the postconfinement phase, as it did in the preconfinement phase.

These results suggest that the difference between groups in performance on the MGT seen in the first four sessions might converge over sessions, such that all groups would show essentially the same level of performance at some point. This convergence was observed only between the radial and antiradial groups in the postconfinement phase for Sessions 9 through $12(z=.39, p>.05-$ two-tailed MannWhitney U test). All other comparisons between groups for Sessions 9 through 12 in both the preand post-confinement phases showed comparable results to those found in Sessions 1 through $4 \mathrm{k}=$ $.41, p>.05$, radial vs. antiradial group in the preconfinement phase; $z=3.13, p<.05$, radial vs. parallel group in the preconfinement phase; $z=3.59$, $p<.05$, antiradial vs. parallel group in the preconfinement phase; $z=5.68, p<.05$, radial vs. parallel group in the postconfinement phase-two-tailed Mann-Whitney U Test).

The improvement in performance on the MGT was paralleled by changes in the pattern of entries. Table 3 presents data on the pattern of entries during the preconfinement phase for Session 12. A chi-square test shows that the differences were not significant $\left[\chi^{2}(4)=1.24, p>.05\right]$. Lambda by columns shows 
Table 3

The Pattern of Entry by Maze During the Preconfinement Phase for Session 12

\begin{tabular}{|c|c|c|c|c|c|c|}
\hline \multirow[b]{3}{*}{ Pattern } & \multicolumn{6}{|c|}{ Mazes } \\
\hline & \multicolumn{2}{|c|}{ Radial } & \multicolumn{2}{|c|}{ Antiradial } & \multicolumn{2}{|c|}{ Parallel } \\
\hline & Obs & Exp & Obs & Exp & Obs & $\operatorname{Exp}$ \\
\hline Typical & 3 & 2.43 & 1 & 2.13 & 3 & 2.43 \\
\hline Unidirectional & 5 & 5.57 & 6 & 4.87 & 5 & 5.57 \\
\hline Immediate Reentry & 0 & 0 & 0 & 0 & 0 & 0 \\
\hline
\end{tabular}

Note-Obs = observed $;$ Exp $=$ expected .

that the reduction in error achieved by knowing what maze the animal was trained in was $0 \%$. Thus, the final pattern of entries shown by the three groups was essentially equivalent in the preconfinement phase. There were no immediate reentries shown by any group, and the number of unidirectional patterns observed was twice that of the number of "typical" patterns.

Table 4 presents data during the postconfinement phase for Session 12. A chi-square test shows that the differences were not significant $\left[\chi^{2}(4)=6.04\right.$, $\mathrm{p}>.05$ ). Lambda by columns shows that the reduction in error was 0\%. Again, as in the preconfinement phase, the final pattern of entries shown by the three groups was essentially equivalent. Unlike the preconfinement phase, however, the number of unidirectional patterns was about one-third the number of "typical" patterns.

The changes in the patterns of entries seen in both phases by Session 12 reflect changes from patterns that were inappropriate to the MGT to patterns that were appropriate to the MGT. In the preconfinement phase, unidirectional and "typical" patterns are appropriate to the MGT, while in the postconfinement phase only a "typical" pattern can be appropriate to the task. An immediate reentry was never appropriate to the MGT. This is reflected in data presented in Tables 3 and 4. Only one animal during Session 12 (in the parallel group during the postconfinement phase) showed an immediate reentry. Unidirectional patterns were predominant only in the preconfinement phase (Table 3) while "typical" patterns were observed in both phases.

Table 4

The Pattern of Entry by Maze During the Postconfinement Phase for Session 12

\begin{tabular}{|c|c|c|c|c|c|c|}
\hline \multirow[b]{3}{*}{ Pattern } & \multicolumn{6}{|c|}{ Mazes } \\
\hline & \multicolumn{2}{|c|}{ Radial } & \multicolumn{2}{|c|}{ Antiradial } & \multicolumn{2}{|c|}{ Parallel } \\
\hline & Obs & Exp & Obs & Exp & Obs & $\operatorname{Exp}$ \\
\hline Typical & 8 & 5.91 & 5 & 5.17 & 4 & 5.91 \\
\hline Unidirectional & 0 & 1.74 & 2 & 1.52 & 3 & 1.74 \\
\hline Immediate Reentry & 0 & 0.35 & $\mathbf{0}$ & 0.30 & 1 & 0.35 \\
\hline
\end{tabular}

Note-Obs = observed $;$ Exp $=$ expected .
In summary, the results showed that the initial patterns of entries produced by the three mazes differed and were as predicted from research on how maze structure affects spontaneous alternation. These differences, in the initial pattern of entries between groups, affected the animals' performance on the MGT. Those groups whose initial pattern of entries was inappropriate to the MGT entered significantly more previously visited goalboxes in the first few sessions than did those groups whose initial pattern of entries was appropriate to the MGT. Significant improvement in performance was seen only in those groups that showed initial patterns of entries that were inappropriate to the MGT. This improvement in performance over sessions was paralleled by changes in the patterns of entries from patterns that were inappropriate to the MGT to patterns that were appropriate to the MGT. These changes in the patterns of entries were such that there was no significant difference between groups for patterns of entries by the last session.

\section{DISCUSSION}

The present experiment examined the effect of maze structure on performance on a MGT. The initial pattern of entries for all three groups was predicted from what is known about how maze structure influences spontaneous alternation. In conditions in which the animal's initial pattern of entries was appropriate to the MGT, initial maze performance was good (e.g., the radial maze during the pre- and postconfinement phases and the antiradial maze in the preconfinement phase). In those conditions in which the animal's initial pattern of entries was inappropriate to the MGT, initial maze performance was poor (e.g., the antiradial maze in the postconfinement phase and the parallel maze in both the preand postconfinement phases).

All groups improved their performances over sessions in both the pre- and postconfinement phases; significant improvement, however, was seen only in groups whose initial performance on the MGT was poor. Those animals with initial patterns that were appropriate to the MGT were already performing the task well and therefore could not improve their performance of the task as much as those whose initial patterns were inappropriate to the MGT. In those groups whose initial pattern of entries was inappropriate to the MGT, improvement entailed changing the pattern of entries to be appropriate to the MGT. Although convergence in performance was seen only between the radial and antiradial groups in the postconfinement phase, it seems likely that had the experiment been carried on for a longer period of time all three groups would have performed the MGT at about the same level in both phases. 
The idea that maze structure affects the animal's performance of a task is not new. Dennis (1935) showed that an animal's initial tendencies in a maze are not random, but follow specific rules. Dennis hypothesized that these initial tendencies affect the animal's performance of a task. Dember and Fowler (1958) and Gaffan and Davies (1981) have more recently hypothesized that the rat's strong tendency to alternate spontaneously under a variety of conditions help the animal to solve a MGT .

The data presented here question the validity of postulating "natural" foraging strategies as an explanation for rats' performance on a MGT. Olton (1979) suggested that a rat's foraging strategy was one of not returning to a place where it had just received reward. For example, Olton and Schlosberg (1978) found that rats had greater difficulty with tasks that required them to return to already visited goalboxes (i.e., win-stay) than with tasks that required them to avoid already visited goalboxes (i.e., win-shift). The results from the Olton and Schlosberg experiment might be better explained in ways other than an appeal to natural foraging strategies. First, the win-stay task might have confused the animals by requiring them to follow two different and contradictory rules in order to solve the task, whereas the win-shift task required the animal only to follow one rule. The win-stay task used by Olton and Schlosberg requires the animal to alternate among four goals, avoiding already visited goals in the preconfinement phase. However, in the postconfinement phase, the win-stay task requires the animal to return to those goals it visited in the preconfinement phase while alternating between goals-so as to avoid revisiting goals it has already visited during the postconfinement phase. The win-shift task, however, requires the animal to avoid already visited goals over both phases; thus, the animals in the win-shift group need apply only one rule consistently in order to solve the task. Second, in both the win-stay and win-shift tasks, animals, over sessions, receive food in all arms of the maze. This reinforcement history should increase the probability that the amimals will enter all arms on any trial, thus favoring a win-shift type of performance. Third, Olton and Schlosberg used a depletable reward and a radial maze in their experiment. As the present experiment and Herrmann et al. (1982) show, both of these factors favor spontaneous alternation, thus favoring performance of the win-shift task. Perhaps Olton and Schlosberg's results would have been different had they used a parallel maze or a nondepletable reward or both.

The overall implications of the present study suggest that environments in which the animal's initial behavior is appropriate to the task will facilitate performance of that task, whereas environments in which the animal's initial behavior is inappropriate to a task will retard performance of that task. The relative efficacy of different contingent situations might be better investigated by appeals to how appropriate the animal's initial behavior is to the reward contingencies (Bolles, 1971; Staddon \& Simmelhag, 1971) than by appeals to innate foraging strategies or innate learning abilities (Olton, 1979; Seligman, 1970).

\section{REFERENCES}

Bolles, R. C. (1970). Species-specific defense reactions and avoidance learning. Psychological Review, 77, 32-48.

Dale, R. H. I. (1982). Parallel-arm maze performance of sighted and blind rats: Spatial memory and maze structure. Behavior Analysis Letters.

Dember, W. N., \& Fowler, H. (1958). Spontaneous alternation behavior. Psychological Bulletin, 55, 412-428.

Dennis, W. (1935). A comparison of the rat's first and second exploration of a maze unit. American Journal of Psychology, 47, 488-490.

Dennis, W., \& Sollenberger, R. T. (1934). Negative adaptation in the maze exploration of rats. Journal of Comparative Psychology, 18, 197-205.

Douglas, R. J., Mitchell, D., \& Kentala, D. (1972). Spontaneous alternation as a function of maze configuration. Psychonomic Science, 27, 285-286.

Gaffan, E. A., \& Davies, J. (1981). The role of exploration in win-shift and win-stay performance on a radial maze. Learning and Motivation, 12, 282-299.

Herrmann, T., Bahr, E., Bremner, B., \& Ellen, P. (1982). Problem solving in the rat: stay vs. shift solutions on the three table task. Animal Learning \& Behavior, 10, 39-45.

JACKSON, M. M. (1941). Reaction tendencies of the white rat in running and jumping situation. Journal of Comparative Psychology, 31, 255-262.

Mueller, J., Schuessler, K., \& Costner, H. (1970). Statistical reasoning in sociology (2nd ed.). Boston: Houghton Mifflin.

Olton, D. S. (1978). Characteristics of spacial memory. In S. H. Hulse, H. Fowler, \& W. K. Honig (Eds.), Cognitive processes in animal behavior. Hillsdale, NJ: Erlbaum.

Olton, D. S. (1979). Mazes, maps, and memory. American Psychologist, 34, 583-596.

Olton, D. S., \& Samuelson, R. L. (1976). Remembrance of places passed: Spatial memory in rats. Journal of Experimental Psychology: Animal Behavior Processes, 2, 97-116.

Olton, D. S., \& Schlosberg, P. (1978). Food searching strategies in young rats: Win-shift predominates over win-stay. Journal of Comparative and Physiological Psychology, 92, 609-618.

Seligman, M. E. P. (1970). On the generality of the laws of learning. Psychological Review, 77, 406-418.

Staddon, J. E. R., \& Simmelhag, V. (1971). The superstition experiment: A reexamination of its implications for the principles of adaptive behavior. Psychological Review, 78, 3-43.

(Manuscript received May 17, 1982; revision accepted for publication September 8, 1983.) 